\title{
Occurrence of Multiple Antibiotic Resistant Bacteria in Aquatic Environments in Central Minnesota
}

\author{
Megan Bollin, Dr. Ellen Jensen, and Dr. David Mitchell* \\ Department of Biology, College of Saint Benedict, St. John's University, Collegeville, MN \\ Student:mebollin@gmail.com \\ Mentors: ejensen@csbsju.edu,dmitchell@csbsju.edu*
}

\begin{abstract}
The purpose of this study was to investigate the possibility that antibiotic resistant bacteria could be isolated and identified in aquatic ecosystems in the lakes on the campus of Saint John's University and the nearby Sauk and Watab Rivers. A total of 125 isolates were collected. Seventy-nine percent of the isolates were gram negative rods. Twenty-six isolates that were resistant to seven or more antibiotics were selected for further investigation. The 26 isolates were all gram negative and members of seven different genera with Flavobacterium and Acinetobacter being the most common. Resistance coefficients were calculated based on optical density values relative to cells grown without antibiotics. Multi-drug resistant, gram negative bacteria were shown to be common in aquatic environments in central Minnesota.
\end{abstract}

\section{INTRODUCTION}

Since the beginning of widespread use of antibiotics in the 1930s and 1940s, pathogenic bacteria have been under selective pressure to evolve mechanisms to limit the effectiveness of antibiotics. As a result the minimum inhibitory concentration (MIC) for antibiotics is slowly increasing. ${ }^{1} \mathrm{MIC}$ is defined as the lowest concentration of antibiotic that inhibits the growth of bacteria and indicates if a particular drug will be effective in treating an infection. Therefore, as time goes on, higher concentrations of antibiotics are required to kill bacteria causing infections. ${ }^{2}$ Unfortunately increased antibiotic exposure will result in even greater selective pressures for evolution to drug resistance via a variety of mechanisms. This is an important human health issue.

Initially antibiotics were only used to treat human infections. Today, antibiotics are used not only to treat infections (human, animal and some plant) but also to promote growth and prevent infections in food animals. It has been estimated that between 35 and 50 million pounds of antibiotics are used per year in the USA. ${ }^{3,4}$ Between $60 \%$ and $80 \%$ of antibiotics used in the USA are used for growth promotion and infection prevention in food animals. ${ }^{5}$ There is increasing awareness that the overuse of antibiotics (agriculturally and clinically) could lead to the evolution of "superbugs" that survive in environments where high concentrations and/or multiple classes of antibiotics are present. Previously, most research on antibiotic resistance has focused on the clinically important pathogenic bacteria causing human infections. ${ }^{6}$ However, over the past decade, it has been observed that bacteria in the environment have the potential to act as a reservoir of antibiotic resistance genes that may be transferred to pathogenic bacteria. ${ }^{7}$ Bacteria can be resistant to one or more antibiotics without being pathogenic. ${ }^{8}$ Therefore, natural environments such as lakes and streams have become targets for antibiotic resistance testing.

Unlike plants and animals - which pass their genes on only to their offspring (vertical gene transfer) - bacteria can transmit genes to other bacteria in the same environment (horizontal gene transfer). Horizontal gene transfer processes include transformation, transduction and conjugation. Since conjugation is not limited to members of the same strain or species, it is the main 
process of horizontal gene transfer in pathogenic bacteria. ${ }^{9}$ Additionally, conjugation also allows the transfer of multiple genes at once. The spread of antibiotic resistance in bacteria through horizontal gene transfer ${ }^{10}$ means that we are losing the ability to treat common bacterial infections simply and effectively. Selective pressure that develops when antibiotics are present in the environment for an extended period of time ${ }^{11}$ will exacerbate this problem. The purpose of this study was to determine if bacteria that are antibiotic resistant are present in waterways surrounding St. John's University (SJU) in central Minnesota. Furthermore we wished to examine the possibility that these bacteria were resistant to multiple classes of antibiotics.

\section{METHODS}

\section{Sample Collection and Initial Plating}

Seventeen different locations were sampled including: the lakes of Saint John's University (Figure 1), the Sauk River in Cold Spring and Watab River in Saint Joseph, Minnesota (Table 1). Water samples $(100 \mathrm{~mL})$ were collected in sterile sampling bottles and placed on ice for transport to the lab. Each sample of water was plated out onto dilution broth $(\mathrm{DB}, 1$ gram tryptone per liter distilled water) agar plates. For each water sample, two spread plates were prepared. One plate was spread with $50 \mu \mathrm{L}$ of the undiluted water sample, the other with $100 \mu \mathrm{L}$ of a 1:10 dilution using dilution broth. These plates were then incubated for 48 hours at room temperature. Roughly ten colonies were selected from each sampling location for additional purification. Each colony selected was transferred using a sterile toothpick onto a new DB agar plate. Plates were incubated at room temperature for 48 hours to allow for growth. If the culture was not pure a streak plate was made on DB agar to separate the mixture. Each pure culture was then streaked onto DB agar so that colony morphology could be determined.

\section{Determination of Antibiotic Resistance}

The effect of eight antibiotics from six classes: streptomycin sulfate, penicillin G, ampicillin, tetracycline, neomycin sulfate, erythromycin, nalidixic acid, and vancomycin on the growth of the 125 isolates was assessed. These antibiotics were selected because each class acts on a slightly different target within the bacterial cell, see Table 2. Isolates were line inoculated onto DB agar plates containing $66 \mu \mathrm{g} / \mathrm{mL}$ of antibiotic using sterile toothpicks. Each plate was inoculated with four isolates, and plates were incubated at room temperature for 48 hours. The amount of growth, color, and swarming capability for each colony was recorded.

The effect of six other antibiotics on the growth of the bacterial isolates was also assessed in experiments in which the antibiotic was present in paper disks (BD/BBL). DB plates were inoculated with the bacteria to create a lawn. Then the six antibiotic disks (cephalothin, sulfamethoxazole/trimethoprim, bacitracin, nitrofurantoin, imipenem, and amoxicillin/clavulanic acid) were placed onto the plate in a circle using sterile forceps and the plates incubated for 48 hours at room temperature. The size of the zone of inhibition was then measured. If an isolate was resistance or sensitive was determined using the table packaged with the antibiotic discs.

Twenty-six isolates that were resistant to seven or more antibiotics in the previous two experiments were chosen for further investigation. In order to determine the concentration range in which these bacterial colonies are susceptible or resistant to the antibiotics, a 96 well plate assay was used. A stock solution of $0.04 \mathrm{~g}$ of antibiotic in $10 \mathrm{~mL}$ of sterile water was created. A serial twofold dilution series using TSB (tryptic soy broth, Difco/BD) was prepared for each antibiotic using the stock solution over the range of 0.25 to $64 \mu \mathrm{g} / \mathrm{mL}$. These antibiotic-TSB mixtures were then pipetted into a standard 96 well plate and inoculated with a $1 \mu \mathrm{L}$ aliquot of an overnight culture of the bacteria, one row was left uninoculated as an antibiotic-only control. The highest concentration tested $(64 \mu \mathrm{g} / \mathrm{mL})$ corresponds: $0.172 \mathrm{mM}$ penicillin $\mathrm{G}, 0.087 \mathrm{mM}$ erythromycin, $0.172 \mathrm{mM}$ 
ampicillin, $0.165 \mathrm{mM}$ amoxicillin, $0.276 \mathrm{mM}$ nalidixic acid, $0.070 \mathrm{mM}$ neomycin sulfate, $0.044 \mathrm{mM}$ streptomycin sulfate, and $0.144 \mathrm{mM}$ tetracycline. The 96 well plates were incubated for 48 hours at room temperature. The optical density $(\mathrm{OD})$ for each well was determined at $600 \mathrm{~nm}$ using a microplate reader (VERSA max, Molecular Devices) and analyzed to compute the resistance coefficient of each of these 26 organisms. Corynebacterium renale was used as a positive control since it is very susceptible to the antibiotics being tested.

Optical Density values were used to compute a numerical resistance coefficient to a particular antibiotic for each isolate. First the OD of the antibiotic-only well was subtracted from the OD of the cell + antibiotic well. This OD difference was then divided by the OD of cell $+0 \mu \mathrm{g} / \mathrm{mL}$ antibiotic well. The resulting resistance coefficient is the amount of growth suppressed by the antibiotic. A resistance coefficient of 1 means that the antibiotic did not inhibit growth. The resistance coefficients were then graphed with the resistance coefficient on the y-axis and the organism isolate on the $\mathrm{x}$-axis. The eight different bars for each organism represent the eight different antibiotic concentrations in order of increasing concentration and the corresponding resistance to that concentration.

The effect of the antibiotics penicillin $\mathrm{G}$ and streptomycin sulfate in combination was also assessed. This combination was selected based on the antibiotics mechanism of action (streptomycin inhibits protein synthesis and penicillin $G$ inhibits cell wall formation) and because the penicillin-aminoglycoside combination is used in introductory microbiology texts as an example of a combination known to have synergism. The antibiotics were tested in a 96 well assay over the range of 0 to $64 \mu \mathrm{g} / \mathrm{mL}$, with both antibiotics having the same concentration (for example, a well would have $0.5 \mathrm{ug} / \mathrm{mL}$ of penicillin $\mathrm{G}$ and $0.5 \mathrm{ug} / \mathrm{mL}$ streptomycin). Resistance coefficients were calculated as in the drug assay.

\section{IDENTIFYING SAMPLES}

The 26 bacteria used in the studies described above were identified using standard microbiological tests. Cellular morphology determined after Gram staining was used to determine which biochemical tests were used in identification. Biochemical tests included: glucose fermentation and nitrate reduction broths, Levine Eosin Methylene Blue (EMB) and Trypticase Soy Agar (TSA) plates, Triple Sugar Iron (TSI) slant, Citrate slant, gelatin deep, and Sulfide Indole Motility (SIM) deep. All test media were incubated at $37^{\circ} \mathrm{C}$ since the bacteria had been shown to grow well at human body temperature. The glucose fermentation was checked after 20 hours and the nitrate reduction was checked after 72 hours. The TSI slant was incubated for 20 hours. SIM deep and gelatin deep were both incubated at $37^{\circ} \mathrm{C}$ for 72 hours. EMB plates and TSA plates were both streaked for isolation and incubated at $37^{\circ} \mathrm{C}$ for 20 hours. ${ }^{12}$ 


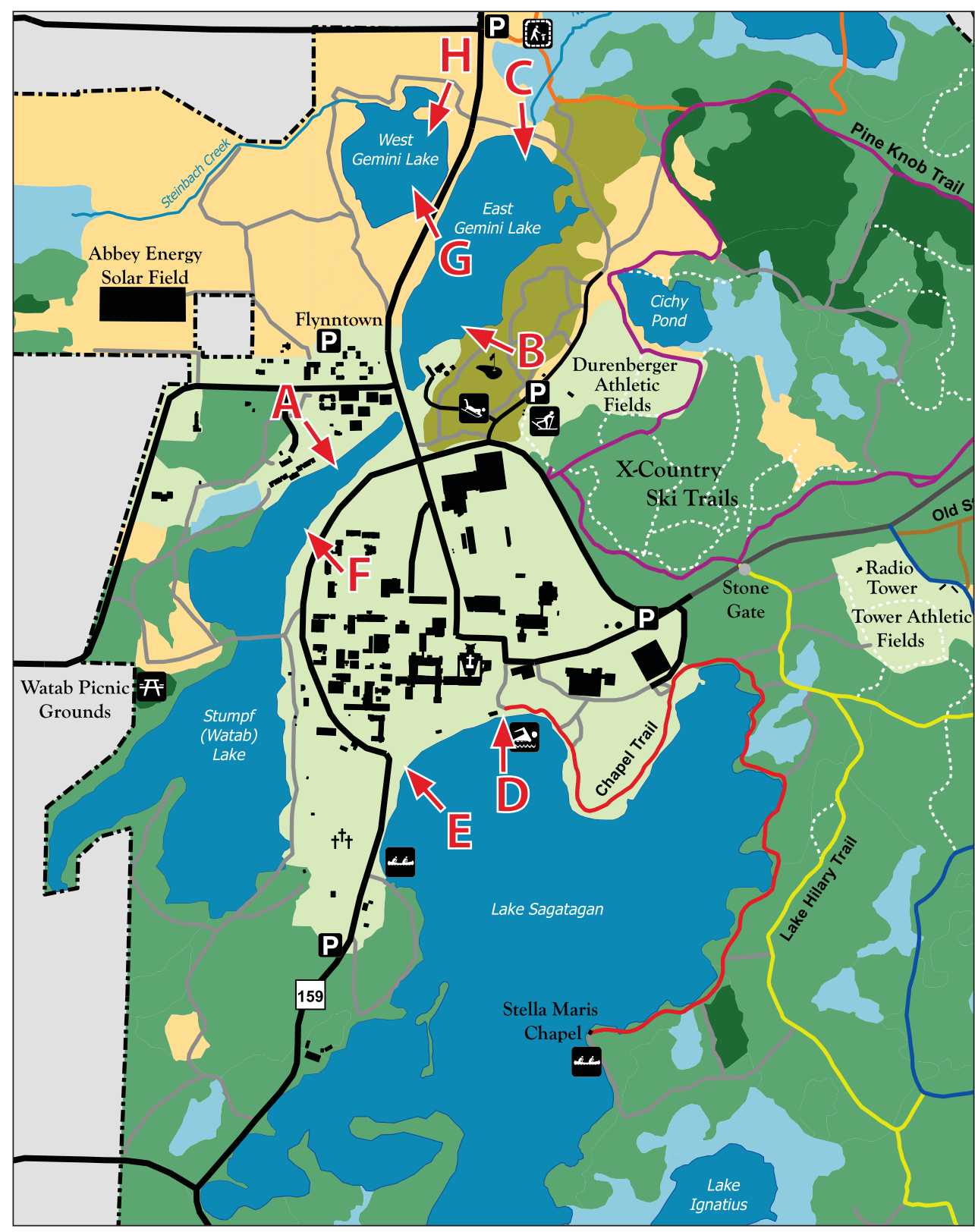

Figure 1. Sampling sites on the St. John's University (SJU) campus. 


\begin{tabular}{|c|c|c|c|c|}
\hline Location & Characteristics & $\begin{array}{l}\text { Sample } \\
\text { Letter }\end{array}$ & Coordinates & Features \\
\hline \multirow{2}{*}{ Stumpf Lake } & \multirow{2}{*}{$\begin{array}{l}\text { Man-made lake created by a } \\
\text { dam in the North Fork of the } \\
\text { Watab River }\end{array}$} & A & $\begin{array}{l}45^{\circ} 35^{\prime} 7^{\prime \prime} \mathrm{N} \\
94^{\circ} 23^{\prime} 43^{\prime} \mathrm{W}\end{array}$ & $\begin{array}{l}\text { Campus Residences } \\
\text { Nearby }\end{array}$ \\
\hline & & $\mathrm{F}$ & $\begin{array}{l}45^{\circ} 34^{\prime} 39^{\prime \prime} \mathrm{N} \\
94^{\circ} 23^{\prime} 40^{\prime \prime} \mathrm{W}\end{array}$ & $\begin{array}{l}\text { Campus Residences } \\
\text { Nearby }\end{array}$ \\
\hline \multirow{2}{*}{ East Gemini Lake } & \multirow{2}{*}{$\begin{array}{l}\text { Man-made lake formed by a } \\
\text { dam in the N. Fork Watab River. } \\
\text { Downstream from Stumpf Lake. }\end{array}$} & B & $\begin{array}{l}45^{\circ} 35^{\prime} 13^{\prime \prime} \mathrm{N} \\
94^{\circ} 23^{\prime} 35^{\prime \prime} \mathrm{W}\end{array}$ & $\begin{array}{l}\text { Where effluent from } \\
\text { SJU Waste Water } \\
\text { Treatment plant enters }\end{array}$ \\
\hline & & $\mathrm{C}$ & $\begin{array}{l}45^{\circ} 35^{\prime} 26^{\prime \prime} \mathrm{N} \\
94^{\circ} 23^{\prime} 21^{\prime \prime} \mathrm{W}\end{array}$ & $\begin{array}{l}\text { Where water flows into } \\
\text { the wetland }\end{array}$ \\
\hline \multirow{2}{*}{ West Gemini Lake } & \multirow{2}{*}{$\begin{array}{l}\text { County Road } 159 \text { separates East } \\
\text { from West Gemini, connected } \\
\text { to East Gemini by } 30 \text { inch } \\
\text { pipe. Water flows from W. to E. } \\
\text { Gemini. }\end{array}$} & G & $\begin{array}{l}45^{\circ} 35^{\prime} 24^{\prime \prime} \mathrm{N} \\
94^{\circ} 23^{\prime} 37^{\prime \prime} \mathrm{W}\end{array}$ & $\begin{array}{l}\text { East side adjacent to } \\
\text { County Road } 159\end{array}$ \\
\hline & & $\mathrm{H}$ & $\begin{array}{l}45^{\circ} 35^{\prime} 24^{\prime \prime} \mathrm{N} \\
94^{\circ} 23^{\prime} 37^{\prime \prime} \mathrm{W}\end{array}$ & $\begin{array}{l}\text { North side adjacent to } \\
\text { farm field }\end{array}$ \\
\hline \multirow{2}{*}{ Lake Sagatagan } & \multirow{2}{*}{$\begin{array}{l}\text { Natural and largest lake on } \\
\text { campus. Surrounded primarily } \\
\text { by forest }\end{array}$} & $\mathrm{D}$ & $\begin{array}{l}45^{\circ} 34^{\prime} 49^{\prime \prime} \mathrm{N} \\
94^{\circ} 23^{\prime} 29^{\prime \prime} \mathrm{W}\end{array}$ & Swimming beach \\
\hline & & $\mathrm{E}$ & $\begin{array}{l}45^{\circ} 34^{\prime} 39^{\prime \prime} \mathrm{N} \\
94^{\circ} 23^{\prime} 40^{\prime \prime} \mathrm{W}\end{array}$ & $\begin{array}{l}\text { Next to monastic } \\
\text { garden }\end{array}$ \\
\hline \multirow{5}{*}{ Sauk River } & \multirow{5}{*}{$\begin{array}{l}\text { Land use within the watershed } \\
\text { is mainly agricultural (small } \\
\text { dairy farms, hay, corn soybeans, } \\
\text { small grains) and mixed decid- } \\
\text { uous forest. The Sauk enters the } \\
\text { Mississippi on the north edge of } \\
\text { St. Cloud. }\end{array}$} & I & $\begin{array}{l}45^{\circ} 27^{\prime} 20^{\prime \prime} \mathrm{N} \\
94^{\circ} 25^{\prime} 22^{\prime \prime} \mathrm{W}\end{array}$ & $\begin{array}{l}\text { Downtown park in } \\
\text { Cold Spring }\end{array}$ \\
\hline & & $\mathrm{J}$ & $\begin{array}{l}45^{\circ} 26^{\prime} 55^{\prime \prime} \mathrm{N} \\
94^{\circ} 25^{\prime} 44^{\prime \prime} \mathrm{W}\end{array}$ & $\begin{array}{l}\text { Fishing Bridge in Cold } \\
\text { Spring }\end{array}$ \\
\hline & & K & $\begin{array}{l}45^{\circ} 27^{\prime} 22^{\prime \prime} \mathrm{N} \\
94^{\circ} 25^{\prime} 21^{\prime \prime} \mathrm{W}\end{array}$ & $\begin{array}{l}\text { Upstream of Chicken } \\
\text { Processing Plant }\end{array}$ \\
\hline & & $\mathrm{L}$ & $\begin{array}{l}45^{\circ} 27^{\prime} 48^{\prime \prime} \mathrm{N} \\
94^{\circ} 24^{\prime} 2^{\prime \prime} \mathrm{W}\end{array}$ & $\begin{array}{l}\text { Downstream from } \\
\text { Processing Plant }\end{array}$ \\
\hline & & M & $\begin{array}{l}45^{\circ} 27^{\prime} 47^{\prime \prime} \mathrm{N} \\
94^{\circ} 24^{\prime} 3^{\prime \prime} \mathrm{W} \\
\end{array}$ & $\begin{array}{l}\text { Downstream from } \\
\text { Processing Plant }\end{array}$ \\
\hline \multirow{4}{*}{ Watab River } & \multirow{4}{*}{$\begin{array}{l}\text { Land use within the watershed } \\
\text { is mainly agricultural (small } \\
\text { dairy farms, hay, corn soybeans, } \\
\text { small grains) and mixed decid- } \\
\text { uous forest. The Watab enters } \\
\text { the Mississippi in Sartell }\end{array}$} & $\mathrm{N}$ & $\begin{array}{l}45^{\circ} 34^{\prime} 10^{\prime \prime} \mathrm{N} \\
94019^{\prime} 42^{\prime \prime} \mathrm{W}\end{array}$ & \multirow{4}{*}{$\begin{array}{l}\text { Millstream Park St. } \\
\text { Joseph. Large park } \\
\text { located at north edge } \\
\text { of the city containing } \\
\text { ball fields, Frisbee } \\
\text { golf course and picnic } \\
\text { grounds }\end{array}$} \\
\hline & & $\mathrm{O}$ & $\begin{array}{c}45^{\circ} 34^{\prime} 11^{\prime \prime} \mathrm{N} \\
94^{\circ} 19^{\prime} 40^{\prime \prime} \mathrm{W} \\
\end{array}$ & \\
\hline & & $\mathrm{P}$ & $\begin{array}{l}45^{\circ} 34^{\prime} 15^{\prime \prime} \mathrm{N} \\
94^{\circ} 19^{\prime} 41^{\prime \prime} \mathrm{W}\end{array}$ & \\
\hline & & Q & $\begin{array}{c}45^{\circ} 34^{\prime} 8^{\prime \prime} \mathrm{N} \\
94^{\circ} 19^{\prime} 44^{\prime \prime} \mathrm{W}\end{array}$ & \\
\hline
\end{tabular}

Table 1. Sampling locations including geographic coordinates and characteristics of the location. 


\begin{tabular}{|c|c|c|c|}
\hline Antibiotic Class & Target & Mode of Action & Effective Against \\
\hline $\begin{array}{c}\text { Aminoglycosides } \\
\text { (Neomycin, Streptomycin) }\end{array}$ & \multirow{3}{*}{ Protein Synthesis } & $\begin{array}{l}\text { Bind irreversibly to } 30 \text { s } \\
\text { ribosomal subunit }\end{array}$ & $\begin{array}{c}\text { Aerobic and facultative } \\
\text { gram negative and gram } \\
\text { positive bacteria }\end{array}$ \\
\hline Tetracycline & & $\begin{array}{l}\text { Bind reversibly to } 30 \mathrm{~s} \\
\text { ribosomal subunit } \\
\text { blocking binding of } \\
\text { aminoacyl-tRNA }\end{array}$ & $\begin{array}{l}\text { Gram positive and gram } \\
\text { negative bacteria }\end{array}$ \\
\hline $\begin{array}{c}\text { Macrolides } \\
\text { (Erythromycin) }\end{array}$ & & $\begin{array}{c}\text { Bind reversibly to } 23 \mathrm{~s} \\
\text { RNA of the } 50 \text { s ribosomal } \\
\text { subunit }\end{array}$ & $\begin{array}{l}\text { Gram positive and gram } \\
\text { negative bacteria }\end{array}$ \\
\hline $\begin{array}{c}\beta \text {-Lactams } \\
\text { (Penicillin G, Ampicillin) }\end{array}$ & \multirow[t]{2}{*}{ Cell Wall Synthesis } & $\begin{array}{l}\text { Irreversibly inhibit cell } \\
\text { wall synthesis by binding } \\
\text { to penicillin-binding } \\
\text { proteins preventing the } \\
\text { final crosslinking of the } \\
\text { peptidoglycan }\end{array}$ & $\begin{array}{c}\text { Gram positive, some gram } \\
\text { negative, and anaerobic } \\
\text { bacteria }\end{array}$ \\
\hline $\begin{array}{l}\text { Glycopeptides } \\
\text { (Vancomycin) }\end{array}$ & & $\begin{array}{c}\text { Inhibit cell wall synthesis } \\
\text { by preventing the addition } \\
\text { of new amino acids to the } \\
\text { peptidoglycan }\end{array}$ & Gram positive bacteria \\
\hline $\begin{array}{c}\text { Quinolone } \\
\text { (Naldixic Acid) }\end{array}$ & DNA Synthesis & Inhibits DNA gyrase & Gram negative bacteria \\
\hline
\end{tabular}

Table 2. Characteristics of the antibiotics used in the initial experiment. ${ }^{13}$

\section{RESULTS}

\section{Antibiotic Resistance}

The majority of isolates (110/125 or 88\%) were resistant to at least one antibiotic (Tables 3-5).

\begin{tabular}{|c|c|c|c|c|c|}
\hline \multirow{2}{*}{$\begin{array}{c}\text { Number of } \\
\text { drugs } \\
\text { resistant to }\end{array}$} & $\begin{array}{c}\text { Sagatagan } \\
(\mathbf{D}+\mathbf{E}) \\
\mathbf{1 5} \text { isolates }\end{array}$ & $\begin{array}{c}\text { Stumpf } \\
(\mathbf{A}+\mathbf{F}) \\
\mathbf{1 5} \text { isolates }\end{array}$ & $\begin{array}{c}\text { East Gemini } \\
(\mathbf{B}+\mathbf{C}) \\
\mathbf{1 5} \text { isolates }\end{array}$ & $\begin{array}{c}\text { West Gemini } \\
\mathbf{( G + H )} \\
\mathbf{1 7} \text { isolates }\end{array}$ & $\begin{array}{c}\text { Total } \\
\mathbf{6 2} \text { isolates }\end{array}$ \\
\hline 0 & 0 & 0 & 1 & 4 & 5 \\
\hline 1 & 2 & 1 & 0 & 1 & 4 \\
\hline 2 & 5 & 1 & 1 & 4 & 11 \\
\hline 3 & 2 & 2 & 3 & 2 & 9 \\
\hline 4 & 4 & 1 & 2 & 1 & 8 \\
\hline 5 & 1 & 3 & 2 & 1 & 7 \\
\hline 6 & 0 & 4 & 2 & 1 & 10 \\
\hline 7 & 0 & 3 & 4 & 3 & 1 \\
\hline All 8 & 1 & 0 & 0 & 0 & 7 \\
\hline
\end{tabular}

Table 3. St. John's lake sample location vs. number of isolates resistant to $\mathrm{X}$ number of drugs. 


\begin{tabular}{|c|c|c|c|c|c|}
\hline \multirow{2}{*}{$\begin{array}{c}\text { Number of } \\
\text { drugs } \\
\text { resistant to }\end{array}$} & $\mathbf{I}$ & $\mathbf{J}$ & $\mathbf{K}$ & $\mathbf{L}$ & M \\
\cline { 2 - 6 } & 0 & 1 & 0 & 0 & 3 \\
\hline 0 & 2 & 1 & 1 & 0 & 1 \\
\hline 1 & 0 & 2 & 4 & 0 & 1 \\
\hline 2 & 2 & 0 & 2 & 1 & 2 \\
\hline 3 & 1 & 3 & 0 & 2 & 0 \\
\hline 4 & 1 & 0 & 0 & 1 & 0 \\
\hline 5 & 1 & 0 & 0 & 0 & 0 \\
\hline 6 & 0 & 0 & 0 & 2 & 0 \\
\hline 7 & 0 & 0 & 0 & 1 & 0 \\
\hline All 8 & & & & & \\
\hline
\end{tabular}

Table 4. Sauk River sampling site vs. number of isolates resistant to X number of drugs.

\begin{tabular}{|c|c|c|c|c|}
\hline \multirow{2}{*}{$\begin{array}{c}\text { Number of } \\
\text { drugs } \\
\text { resistant to }\end{array}$} & $\mathbf{N}$ & $\mathbf{O}$ & $\mathbf{P}$ & $\mathbf{Q}$ \\
\cline { 2 - 5 } & 1 & 1 & 2 & 2 \\
\hline 0 & 2 & 0 & 1 & 1 \\
\hline 1 & 1 & 1 & 1 & 4 \\
\hline 2 & 1 & 2 & 3 & 0 \\
\hline 3 & 1 & 2 & 0 & 0 \\
\hline 4 & 0 & 1 & 0 & 0 \\
\hline 5 & 0 & 0 & 0 & 0 \\
\hline 6 & 1 & 0 & 0 & 0 \\
\hline 7 & 0 & 0 & 0 & 0 \\
\hline All 8 & & & 0 & \multicolumn{2}{|c|}{ Watab River } \\
\hline
\end{tabular}

Table 5. Watab River sampling site vs. number of isolates resistant to X number of drugs.

The Fisher Exact Test ${ }^{14}$ was used to determine if the differences in the number of isolates resistant to $\mathrm{X}$ drugs between sites was significant. The $\mathrm{P}$ values were as follows: comparison of just the four SJU lakes $p=0.20$; comparison of five Sauk River sites $p=0.020$; comparison of four Watab River sites $\mathrm{p}=0.37$. The Fisher Exact Test was also used to compare the regions SJU lakes, Sauk River and Watab River, $\mathrm{p}=0.022$.

A data analysis using the Fisher Exact Test of the 125 isolates was also made to see if there were differences in the pattern of resistance to the different drug targets and classes for each water source (Tables 7-9). The St. John's Lakes, Sauk River and Watab River were also compared to each other using the Fisher Exact Test (Table 10). 


\begin{tabular}{|c|c|c|c|}
\hline \multirow{2}{*}{$\begin{array}{c}\text { Number of drugs } \\
\text { isolate was resistant }\end{array}$} & $\begin{array}{c}|c| \\
\text { SJU Lakes } \\
\text { (62 isolates) }\end{array}$ & $\begin{array}{c}\text { Sauk River } \\
\text { (35 isolates) }\end{array}$ & $\begin{array}{c}\text { Watab River } \\
\text { (28 isolates) }\end{array}$ \\
\cline { 2 - 4 } & 5 & 4 & 6 \\
\hline 0 & 4 & 5 & 4 \\
\hline 1 & 11 & 7 & 7 \\
\hline 2 & 9 & 7 & 6 \\
\hline 3 & 8 & 6 & 3 \\
\hline 4 & 7 & 2 & 1 \\
\hline 5 & 7 & 1 & 0 \\
\hline 6 & 10 & 2 & 1 \\
\hline 7 & 1 & 1 & 0 \\
\hline All 8 & & & \\
\hline
\end{tabular}

Table 6. Comparison of the number of isolates resistant to $\mathrm{X}$ number of drugs between SJU Lakes, Sauk River, and Watab River.

\begin{tabular}{|c|c|c|c|c|c|c|c|}
\hline \multirow[b]{2}{*}{$\begin{array}{l}\text { Antibiotic } \\
\text { Class }\end{array}$} & \multirow[b]{2}{*}{$\begin{array}{c}\text { Antibiotic } \\
\text { Target }\end{array}$} & \multicolumn{4}{|c|}{ Lake sampled } & \multirow[b]{2}{*}{$\begin{array}{l}\text { Fisher } \\
\text { p value } \\
\text { for } \\
\text { Target }\end{array}$} & \multirow[b]{2}{*}{$\begin{array}{l}\text { Fisher } \\
\text { p value } \\
\text { for } \\
\text { Class }\end{array}$} \\
\hline & & $\begin{array}{l}\text { Sagatagan } \\
(\mathrm{D}+\mathrm{E}) \\
15 \text { isolates }\end{array}$ & $\begin{array}{c}\text { Stumpf } \\
(\mathrm{A}+\mathrm{F}) \\
15 \text { isolates }\end{array}$ & $\begin{array}{c}\text { East } \\
\text { Gemini } \\
(B+C) \\
15 \text { isolates }\end{array}$ & $\begin{array}{c}\text { West } \\
\text { Gemini } \\
(\mathrm{G}+\mathrm{H}) \\
17 \text { isolates }\end{array}$ & & \\
\hline $\begin{array}{l}\text { Aminoglycosides } \\
\text { (Neomycin, } \\
\text { Streptomycin) }\end{array}$ & \multirow{3}{*}{$\begin{array}{l}\text { Protein } \\
\text { Synthesis }\end{array}$} & 15 & 15 & 13 & 13 & \multirow{3}{*}{0.057} & 0.057 \\
\hline Tetracycline & & 1 & 0 & 1 & 0 & & 0.596 \\
\hline $\begin{array}{c}\text { Macrolides } \\
\text { (Erythromycin) }\end{array}$ & & 5 & 9 & 9 & 6 & & 0.2515 \\
\hline $\begin{array}{l}\beta \text {-Lactams } \\
\text { (Penicillin G, } \\
\text { Ampicillin) }\end{array}$ & \multirow{3}{*}{$\begin{array}{l}\text { Cell Wall } \\
\text { Synthesis }\end{array}$} & 5 & 11 & 10 & 6 & \multirow{3}{*}{0.089} & 0.052 \\
\hline Glycopeptides & & & & & & & \multirow{2}{*}{0.197} \\
\hline (Vancomycin) & & 11 & 12 & 13 & 9 & & \\
\hline $\begin{array}{c}\text { Quinolone } \\
\text { (Naldixic Acid) }\end{array}$ & $\begin{array}{c}\text { DNA } \\
\text { Synthesis }\end{array}$ & 2 & 5 & 5 & 5 & 0.572 & 0.572 \\
\hline
\end{tabular}

Table 7. SJU Lake vs. number of isolates with resistance based on drug target and drug class. 


\begin{tabular}{|c|c|c|c|c|c|c|c|c|}
\hline \multirow[b]{2}{*}{$\begin{array}{l}\text { Antibiotic } \\
\text { Class }\end{array}$} & \multirow[b]{2}{*}{$\begin{array}{l}\text { Antibiotic } \\
\text { Target }\end{array}$} & \multicolumn{5}{|c|}{ Sauk River Sample } & \multirow{2}{*}{$\begin{array}{l}\text { Fisher } \\
\text { p value } \\
\text { for } \\
\text { Target }\end{array}$} & \multirow{2}{*}{$\begin{array}{l}\text { Fisher } \\
\text { p value } \\
\text { for } \\
\text { Class }\end{array}$} \\
\hline & & $\begin{array}{c}\text { I } \\
7 \text { isolates }\end{array}$ & $\begin{array}{c}\mathrm{J} \\
7 \text { isolates }\end{array}$ & $\begin{array}{c}\mathrm{K} \\
7 \text { isolates }\end{array}$ & $\begin{array}{c}\mathrm{L} \\
7 \text { isolates }\end{array}$ & $\begin{array}{c}\mathrm{M} \\
7 \text { isolates }\end{array}$ & & \\
\hline $\begin{array}{c}\text { Aminoglycosides } \\
\text { (Neomycin, } \\
\text { Streptomycin) }\end{array}$ & \multirow{3}{*}{$\begin{array}{l}\text { Protein } \\
\text { Synthesis }\end{array}$} & 4 & 5 & 4 & 4 & 1 & \multirow{3}{*}{0.118} & 0.0018 \\
\hline Tetracycline & & 0 & 0 & 0 & 1 & 0 & & 1.00 \\
\hline $\begin{array}{c}\text { Macrolides } \\
\text { (Erythromycin) }\end{array}$ & & 2 & 0 & 0 & 5 & 0 & & 0.0014 \\
\hline $\begin{array}{l}\beta \text {-Lactams } \\
\text { (Penicillin G, } \\
\text { Ampicillin) }\end{array}$ & \multirow{2}{*}{$\begin{array}{l}\text { Cell Wall } \\
\text { Synthesis }\end{array}$} & 7 & 4 & 3 & 7 & 3 & 0.056 & 0.018 \\
\hline $\begin{array}{l}\text { Glycopeptides } \\
\text { (Vancomycin) }\end{array}$ & & 2 & 3 & 3 & 6 & 3 & & 0.329 \\
\hline $\begin{array}{c}\text { Quinolone } \\
\text { (Naldixic Acid) }\end{array}$ & $\begin{array}{c}\text { DNA } \\
\text { Synthesis }\end{array}$ & 1 & 0 & 0 & 4 & 0 & 0.015 & 0.015 \\
\hline
\end{tabular}

Table 8. Sauk River samples vs. number of isolates with resistance based on drug target and drug class.

\begin{tabular}{|c|c|c|c|c|c|c|c|}
\hline \multirow{2}{*}{$\begin{array}{l}\text { Antibiotic } \\
\text { Class }\end{array}$} & \multirow{2}{*}{$\begin{array}{c}\text { Antibiotic } \\
\text { Target }\end{array}$} & \multicolumn{4}{|c|}{ Watab River Sample } & \multirow{2}{*}{$\begin{array}{c}\text { Fisher } \\
\text { p value for } \\
\text { Target }\end{array}$} & \multirow{2}{*}{$\begin{array}{l}\text { Fisher } \\
\text { p value for } \\
\text { Class }\end{array}$} \\
\hline & & $\begin{array}{c}\mathrm{N} \\
7 \text { isolates }\end{array}$ & $\begin{array}{c}0 \\
7 \text { isolates }\end{array}$ & $\begin{array}{c}P \\
7 \text { isolates }\end{array}$ & $\begin{array}{c}\mathrm{Q} \\
7 \text { isolates }\end{array}$ & & \\
\hline $\begin{array}{c}\text { Aminoglycosides } \\
\text { (Neomycin, } \\
\text { Streptomycin) }\end{array}$ & \multirow{3}{*}{$\begin{array}{l}\text { Protein } \\
\text { Synthesis }\end{array}$} & 5 & 4 & 3 & 2 & \multirow{3}{*}{0.60} & 0.596 \\
\hline Tetracycline & & 1 & 0 & 0 & 0 & & 1.00 \\
\hline $\begin{array}{c}\text { Macrolides } \\
\text { (Erythromycin) }\end{array}$ & & 1 & 2 & 0 & 0 & & 0.58 \\
\hline $\begin{array}{l}\beta \text {-Lactams } \\
\text { (Penicillin G, } \\
\text { Ampicillin) }\end{array}$ & \multirow{2}{*}{$\begin{array}{l}\text { Cell Wall } \\
\text { Synthesis }\end{array}$} & 4 & 5 & 0 & 3 & \multirow{2}{*}{0.81} & 0.044 \\
\hline $\begin{array}{l}\text { Glycopeptides } \\
\text { (Vancomycin) }\end{array}$ & & 2 & 4 & 5 & 1 & & 0.17 \\
\hline $\begin{array}{c}\text { Quinolone } \\
\text { (Naldixic Acid) }\end{array}$ & $\begin{array}{c}\text { DNA } \\
\text { Synthesis }\end{array}$ & 0 & 0 & 0 & 0 & 1.00 & 1.00 \\
\hline
\end{tabular}

Table 9. Watab River samples vs. number of isolates with resistance based on drug target and drug class. 


\begin{tabular}{|c|c|c|c|c|c|c|}
\hline \multirow[b]{2}{*}{$\begin{array}{l}\text { Antibiotic } \\
\text { Class }\end{array}$} & \multirow[b]{2}{*}{$\begin{array}{c}\text { Antibiotic } \\
\text { Target }\end{array}$} & \multicolumn{3}{|c|}{ Lake Sampled } & \multirow{2}{*}{$\begin{array}{l}\text { Fisher } \\
\text { p value for } \\
\text { Target }\end{array}$} & \multirow{2}{*}{$\begin{array}{c}\text { Fisher } \\
\text { p value for } \\
\text { Class }\end{array}$} \\
\hline & & $\begin{array}{l}\text { SJU Lakes } \\
62 \text { isolates }\end{array}$ & $\begin{array}{l}\text { Sauk river } \\
35 \text { isolates }\end{array}$ & $\begin{array}{c}\text { Watab River } \\
28 \text { isolates }\end{array}$ & & \\
\hline $\begin{array}{l}\text { Aminoglycoside } \\
\text { (Neomycin, } \\
\text { Streptomycin) }\end{array}$ & \multirow{3}{*}{$\begin{array}{l}\text { Protein } \\
\text { Synthesis }\end{array}$} & 56 & 17 & 14 & \multirow{3}{*}{$<0.001$} & $<0.001$ \\
\hline Tetracycline & & 2 & 1 & 1 & & 1.00 \\
\hline $\begin{array}{c}\text { Macrolides } \\
\text { (Erythromycin) }\end{array}$ & & 29 & 7 & 3 & & $<0.001$ \\
\hline $\begin{array}{l}\beta \text {-Lactams } \\
\text { (Penicillin G, } \\
\text { Ampicillin) }\end{array}$ & \multirow{2}{*}{$\begin{array}{l}\text { Cell Wall } \\
\text { Synthesis }\end{array}$} & 32 & 24 & 12 & \multirow{2}{*}{0.37} & 0.16 \\
\hline $\begin{array}{l}\text { Glycopeptides } \\
\text { (Vancomycin) }\end{array}$ & & 45 & 17 & 12 & & 0.009 \\
\hline $\begin{array}{c}\text { Quinolone } \\
\text { (Naldixic Acid) }\end{array}$ & $\begin{array}{c}\text { DNA } \\
\text { Synthesis }\end{array}$ & 17 & 5 & 0 & .002 & 0.002 \\
\hline
\end{tabular}

Table 10. Comparison between the three regions (St. John's Lakes, Sauk River and Watab River) vs. number of isolates with resistance based on drug target and drug class.

In the antibiotic disc assay $26 \%$ of the isolates were sensitive to all of the drugs (cephalothin, sulfamethoxazole/trimethoprim, bacitracin, nitrofurantoin, imipenem, and amoxicillin/clavulanic acid) tested, and $74 \%$ were resistant to at least one of the 6 drugs. None of the isolates were resistant to imipenem. Only the St. John's Lake samples $(p=0.016)$ had a Fisher Exact Test $p$ value that indicated a significant difference between samples for the number of drugs the isolates were resistant to. Table 11 shows the clinical dosage and resulting serum concentration of the antibiotics used in the 96 well assay. All of the drug's peak serum concentration would be within the concentration range used in the 96 well assay $(0.5-64 \mu \mathrm{g} / \mathrm{mL})$.

\begin{tabular}{llcc}
\hline \multicolumn{1}{c}{ Antibiotic } & \multicolumn{1}{c}{ Common Dosage } & $\begin{array}{c}\text { Peak Serum } \\
\text { Concentration }\end{array}$ & Concentration (mM) \\
\hline Amoxicillin & $250 \mathrm{mg}$ per 8 hours & $5 \mu \mathrm{g} / \mathrm{mL}$ & $0.014 \mathrm{mM}$ \\
\hline Ampicillin & $250-500 \mathrm{mg}$ per 6 hours & $3 \mu \mathrm{g} / \mathrm{mL}$ & $0.009 \mathrm{mM}$ \\
\hline Streptomycin Sulfate & $1 \mathrm{~g}$ a day & $25-50 \mu \mathrm{g} / \mathrm{mL}$ & $0.026 \mathrm{mM}$ \\
\hline Nalidixic Acid & 4 g per day & $20-40 \mu \mathrm{g} / \mathrm{mL}$ & $0.172 \mathrm{mM}$ \\
\hline Penicillin G & 1 million units & $14.4 \mu \mathrm{g} / \mathrm{mL}$ & $0.043 \mathrm{mM}$ \\
\hline Erythromycin & $\begin{array}{l}250 \text { or } 500 \mathrm{mg} \\
\text { N times a day }\end{array}$ & $7.21 \mu \mathrm{g} / \mathrm{mL}$ & $0.010 \mathrm{mM}$ \\
\hline Neomycin Sulfate & $4 \mathrm{~g}$ a day & $6.1 \mu \mathrm{g} / \mathrm{mL}$ & $0.010 \mathrm{mM}$ \\
\hline Vancomycin & $1 \mathrm{~g}$ per 12 hours & $20 \mu \mathrm{g} / \mathrm{mL}$ & $0.014 \mathrm{mM}$ \\
Tetracycline & $\begin{array}{l}125 \text { to } 500 \mathrm{mg} \\
\text { per } 12 \text { hours }\end{array}$ & $4-5 \mu \mathrm{g} / \mathrm{mL}$ & $0.011 \mathrm{mM}$ \\
\hline
\end{tabular}

Table 11. Clinical dosage and peak serum concentration of each antibiotic and the corresponding $\mathrm{mM}$ concentration for each antibiotic used in the 96 well assay. 
The data from the 96 well antibiotic assays on the 26 isolates were analyzed based on resistance coefficients. ${ }^{8}$ Organisms with coefficients greater than 0.8 were considered resistant to the drug at the concentration being tested. ${ }^{15}$ Graphs of the resistance coefficient data for each concentration tested showed three distinct patterns: 1) the organism's resistance coefficient was between $0.8-1$ for all concentrations tested; 2) the organism was inhibited at higher antibiotic concentrations; and 3) at some concentrations the antibiotic caused increased growth and a resistance coefficient greater than 1 (Figure 2). Table 12 shows the distribution between the three patterns for the different antibiotics.

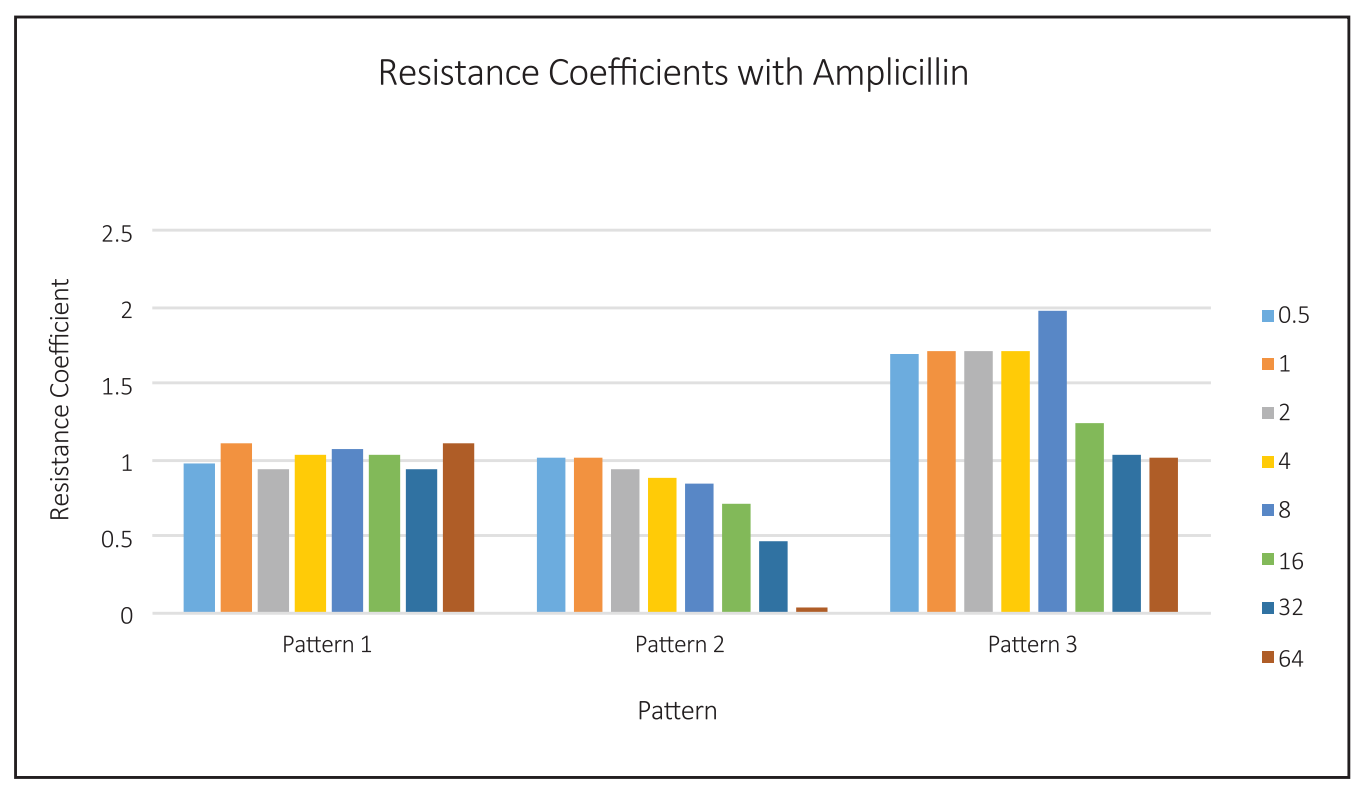

Figure 2. Resistance coefficient patterns for $0.5 \mu \mathrm{g} / \mathrm{mL}$ to $64 \mu \mathrm{g} / \mathrm{mL}$ antibiotic using ampicillin as an example.

\begin{tabular}{|c|c|c|c|c|c|c|c|c|}
\hline $\begin{array}{c}\text { Number } \\
\text { of isolates } \\
\text { with } \\
\text { pattern }\end{array}$ & 泀 & 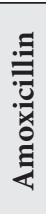 & 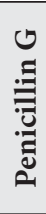 & 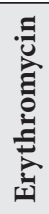 & 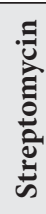 & 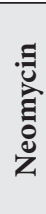 & & 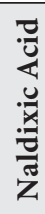 \\
\hline Pattern 1 & 17 & 18 & 19 & 19 & 17 & 15 & 0 & 19 \\
\hline Pattern 2 & 2 & 4 & 2 & 5 & 2 & 11 & 26 & 7 \\
\hline Pattern 3 & 6 & 3 & 4 & 2 & 6 & 0 & 0 & 0 \\
\hline
\end{tabular}

Table 12. Growth patterns in the 96 well plates. Pattern $1=$ Resistant at all concentrations tested,

Pattern 2 = Growth inhibited at higher concentrations, Pattern 3 = Increased growth at some concentrations

Eight of the isolates (A1, A3, A11, C6, C10, H1b, L7, N2) were resistant at all concentrations (0.5-64 $\mu \mathrm{g} / \mathrm{mL}$ ) to penicillin $\mathrm{G}$ and streptomycin in the 96 well assay. These were selected for use in a combined penicillin G plus streptomycin 96 well assay. After incubation the resistance coefficients for each well were calculated in a similar manner as in the previous experiment. All isolates showed synergism between the two drugs (Figure 3) in that higher concentrations caused an inhibition in growth. Resistance coefficients of 0.2 or less are required for successful treatment. ${ }^{15}$ Peak serum concentrations are: streptomycin $25-50$ $\mu \mathrm{g} / \mathrm{mL}$ and penicillin G14.4 $\mu \mathrm{g} / \mathrm{mL}$. Two of the isolates have MICs that are low enough that the combination would be successful in treating an infection: $\mathrm{All}$ with $\mathrm{MIC}=4$ and $\mathrm{L7}$ with $\mathrm{MIC}=8$. The other six isolates would require penicillin $\mathrm{G}$ concentrations $>14.4 \mu \mathrm{g} / \mathrm{mL}$ which are not normally achieved in serum. 
Gram Stain Results of the 125 isolates

The majority of the isolates were gram negative rods (79\%) with the next most frequent morphology being gram positive endospore forming rods (8\%). The remaining isolates were subdivided between: gram positive non-endospore forming rods, cocci, gram variable rods. A Fisher Exact Test was performed in order to determine if cellular morphology was dependent on location sampled; the $\mathrm{p}$ value of 0.36 indicates the differences were not significant.

\section{Identification of the 26 Isolates:}

The two most common genera were Acinetobacter and Flavobacterium. Seven out of 26 were classified as Acinetobacter (A1, B7, C10, F3b, H3b, L6, N2) and eight as Flavobacterium (A2, A3, A6, A7, A11, C1, C6, G3). The other genera represented in these 26 isolates were Enterobacter with five isolates, Pseudomonas aeruginosa with two isolates, Proteus with one isolate, Klebsiella with one isolate, and Salmonella with one isolate. One isolate would not grow and was presumed to have died.

The Acinetobacter and Flavobacterium isolates resistance to the antibiotics that target protein and cell wall synthesis were compared (Table 13) to see if they had the same pattern. Acinetobacter isolates A1, C10 and L6 have the same pattern. Flavobacterium isolates A2, A3, and C6 have the same pattern.

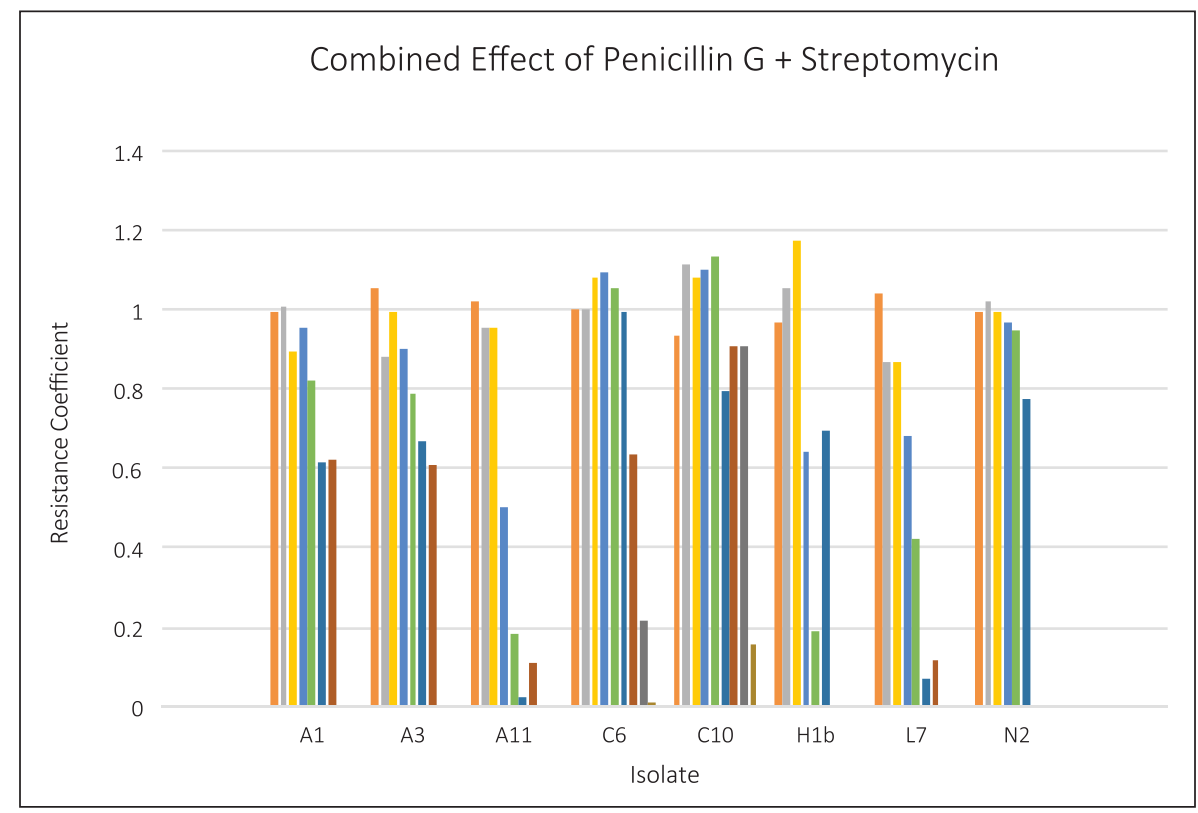

Figure 3. Combined effect of $0.25 \mathrm{ug} / \mathrm{mL}$ to $64 \mathrm{ug} / \mathrm{mL}$ penicillin $\mathrm{G}$ and streptomycin on eight isolates. 


\begin{tabular}{|l|c|c|c|c|c|c|c|c|}
\hline & \multicolumn{3}{|c|}{ Cell Wall Disruption Antibiotics } & \multicolumn{3}{c|}{ Protein Synthesis Disruption Antibiotics } \\
\hline $\begin{array}{l}\text { Acinetobacter } \\
\text { Isolates }\end{array}$ & Pen. G & Amp. & Amox. & Vanc. & Neo. & Strep. & Tet. & Eryth. \\
\hline A1 & + & + & + & + & + & + & - & + \\
\hline B7 & + & + & + & + & - & + & - & + \\
\hline C10 & + & + & + & + & + & + & - & + \\
\hline F3B & + & - & + & + & + & + & - & + \\
\hline H3B & - & - & - & + & + & - & - & - \\
\hline L6 & + & + & + & + & + & + & - & + \\
\hline N2 & + & - & + & + & + & + & - & - \\
\hline Flavobacterim & & & & & & & & \\
Isolates & + & + & + & + & + & + & - & + \\
\hline A2 & + & + & + & + & + & + & - & + \\
\hline A3 & + & + & + & + & - & - & - & + \\
\hline A6 & + & + & - & + & - & + & - & + \\
\hline A7 & - & + & + & + & - & + & - & + \\
\hline A11 & + & + & - & + & - & - & - & + \\
\hline C1 & + & + & + & + & + & + & - & + \\
\hline C6 & - & - & + & + & - & - & + \\
\hline G3 & + & + & + & + & + & + & + \\
\hline
\end{tabular}

Table 13. Resistance to different antibiotics for the Acinetobacter and Flavobacterium isolates. Symbols: $+=$ resistant and $-=$ susceptible.

\section{DISCUSSION}

Previous studies show that the frequency of resistant bacteria in fresh water is variable and ranges from: $18.6 \%$ to $20.4 \%$ for isolates from Oregon streams and rivers to $92 \%$ for rural ground water in West Virginia. ${ }^{16-18}$ One would predict that samples collected from rural central Minnesota lakes and streams would have some antibiotic resistant bacteria present but that the frequency would be low due to the absence of a large dense population. Instead our samples had a high percentage (88\%) of bacteria resistant to at least one of eight antibiotics (Table 6). The Fisher Exact Test showed that the number of resistant isolates for the SJU lakes and Watab River samples were not significantly different (Tables 3 and 5). There was, however, a significant difference between the five Sauk River samples. Sample L which was downstream from a chicken processing plant had a higher frequency of isolates that were resistant to 6 or more drugs (Table 4). When comparing the three regions using the Fisher Exact Test $(\mathrm{p}=0.022)$ the SJU lakes were shown to have a significantly higher number of isolates resistant to five or more drugs than the Sauk and Watab Rivers. This suggests that distinct microenvironments for horizontal gene transfer may be present in the waterways.

The antibiotic resistance data from the 125 isolates was analyzed using the Fisher Exact Test to determine if there were differences in the pattern of resistance to the different drug targets and classes either within or between the three regions (Tables 7-10). The differences seen for tetracycline resistance were not significant either within a region or between the three regions. Aminoglycoside resistance was significantly different when comparing the Sauk River samples ( $M$ had decreased number of resistant isolates), close to being significant for the SJU lake samples (decreased number of resistant isolates for West Gemini) and when the three regions were compared the SJU lakes had significantly higher number of resistant isolates than the samples from the two rivers. Differences for Erythromycin/macrolides were significant both within the Sauk River samples (increased for L) 
and between the three regions (increased for the SJU lakes). Resistance to protein synthesis inhibitors was significantly different between the regions (increased for SJU lakes). $\beta$-lactam resistance was significantly different within both the Watab River samples (decreased for sample P) and Sauk River (increased samples I and L), and almost significant within the SJU lake samples (decreased numbers for Sagatagan and West Gemini) but differences were not significant when the three regions were compared. Vancomycin/glycopeptide resistance was not significantly different when the samples within a region were compared but was significantly different when the three regions were compared to each other, in that the SJU lakes had an increased number of resistant isolates. Cell wall synthesis inhibitor differences both within and between regions were not significant. Nalidixic acid/quinolone resistance differences were only significant for the Sauk River locations and sample $\mathrm{L}$ had larger numbers of resistant isolates than the other four locations.

Two patterns of antibiotic resistance were expected to be seen in the 96 well antibiotic assays: resistant at all concentrations tested (Pattern 1) and sensitive at higher concentrations (Pattern 2). A majority of the 26 isolates acted as predicted. However, $23 \%$ of the isolates exhibited a third pattern (increased growth at some concentrations of the drug) with some drugs (ampicillin, amoxicillin, penicillin G, erythromycin, streptomycin) as shown in Figure 2. Given that antibiotics function to block the synthesis of molecules required for growth, seeing Pattern 3 seemed improbable. A search of the literature determined that Pattern 3 has been previously seen with gram negative bacteria isolated from soil especially members of the genera Pseudomonas and Burkholderia ${ }^{19}$ and Salmonella. ${ }^{20}$ This phenomenon has been named subsistence ${ }^{19,20}$ and this work provides the first evidence that this phenomenon can occur in fresh water aquatic environments. It would be interesting to see if the isolates exhibiting Pattern 3 are also able to use some antibiotics as a sole carbon source like the Salmonella isolates can. ${ }^{20}$

The most frequent bacterial cell morphology seen from all of the locations sampled was gram negative rods (79\%) followed by gram positive endospore-forming rods (8\%). The Fisher Exact Test showed the variation between locations and morphologies was not significant. Previous studies have shown that these two morphologies are the most prevalent in fresh water lakes, streams and rivers. ${ }^{16,21,24}$

In the 26 isolates chosen for identification and further study the most common genera isolated were: 31\% Flavobacterium, 27\% Acinetobacter, 19\% Enterobacter, 8\% Pseudomonas, and 4\% each for Proteus, Klebsiella, and Salmonella. Previous studies have also found these genera in fresh water but the actual percentages present varies from study to study. The percentages of Acinetobacter from our samples (27\%) are similar to those found in unpolluted lakes, streams and rivers ${ }^{21,23}$ but higher than that found in polluted streams and rivers. ${ }^{23,}{ }^{24}$ The percentages of Pseudomonas (8\%) are similar to those found for Lake Michigan and streams in Oregon ${ }^{21,24}$ but much lower than those seen in samples collected from rivers in Estonia or Oregon. ${ }^{8,16,23}$ None of the samples contained Escherichia coli and contained much lower numbers of Enterobacteriaceae than all of the other studies. Our Flavobacterium percentage (31\%) was much higher than was reported in Oregon streams, Estonia rivers and the Great Lakes ${ }^{8,24}$ but also much lower than that of polluted rivers. ${ }^{23}$

Eighty-six percent of the Acinetobacter isolates are resistant to five or more drugs and to at least one member of three or more antibiotic classes. Seventy-five percent of the Flavobacterium isolates are also resistant to five or more drugs and to at least one member of three or more antibiotic classes. Multiple Drug Resistant (MDR) is defined as being resistant to one or members of at least three classes of antibiotics. ${ }^{25}$ Pseudomonas aeruginosa, most of the other species of Pseudomonas and many other genera of gram negative bacteria have a natural resistance to penicillin $G$ because the drug cannot get through their outer membrane; so observing a high frequency of resistance to this drug in isolates that are gram negative rods would be expected. Fifty-seven percent of the Acinetobacter isolates and $87.5 \%$ of the Flavobacterium isolates were resistant to ampicillin. Other studies have also shown high levels of resistance to ampicillin: 90\% of Pseudomonas isolates were resistant, $100 \%$ of Acinetobacter Abc but less than $1 \%$ of environmental Acinetobacter species were resistant, and $73 \%$ of Flavobacterium were resistant. ${ }^{23,26,27}$ If we look at the drugs that most of the 
isolates were sensitive to (tetracycline and imipenem) we see that other studies also saw no-tolow frequencies of resistance for environmental isolates of these three genera. ${ }^{23},{ }^{25}$ All three genera (Acinetobacter, Flavobacterium and Pseudomonas) are known for: their metabolic versatility especially for carbon sources utilized, ability to cope with environmental stress, large genomes and proven ability for horizontal gene transfer. ${ }^{25,26,28,29}$

Acinetobacter, Flavobacterium and Pseudomonas are genera for whom the normal habitat of most species is fresh water. The three genera also contain some species that are recognized as being opportunistic pathogens causing human infections, especially nosocomial infections. Pseudomonas aeruginosa has long been known to cause opportunist infections but the Acinetobacter $A b c$ complex has only emerged over the last 20 years as being an important cause of nosocomial infections. ${ }^{30}$ Pseudomonas aeruginosa and the Acinetobacter Abc complex are responsible for causing more than $80 \%$ of non-enteric gram negative rod infections in humans. ${ }^{31}$ It would be interesting to key out the Acinetobacter and Flavobacterium isolates to see if they are species that are strictly environmental or human associated and opportunistic pathogen like the Acinetobacter $A b c$ complex.

In conclusion this study demonstrates that antibiotic resistant bacteria can be isolated from a number of water sources on our campus and in the local community. The majority of these bacteria would be expected to be present in these waterways yet the level of resistance to antibiotics at clinical concentrations (and above) and resistance to multiple classes of antibiotics is disconcerting. In addition our data indicates that patterns of antibiotic resistance can be quite different in nearby waterways. Taken together with the works cited in this paper questions about the (over) use of antibiotics in agriculture and medicine may need to be addressed much more critically in the near future.

\section{REFERENCES}

[1] Fernandez, L., Breidenstein, E., and Hancock, R. (2012) Importance of Adaptive and Stepwise Changes in the Rise and Spread of Antimicrobial Resistance, John Wiley \& Sons, Inc., Hoboken, New Jersey.

[2] Martinez, J. L. (2008) Antibiotics and antibiotic resistance genes in natural environments, Science $321,365-367$.

[3] Davies, J., and Davies, D. (2010) Origins and evolution of antibiotic resistance, Microbiology and Molecular Biology Reviews : MMBR 74, 417-433.

[4] Shea, K. M. (2003) Antibiotic resistance: what is the impact of agricultural uses of antibiotics on children's health?, Pediatrics 112, 253-258.

[5] Silbergeld, E. K., Graham, J., and Price, L. B. (2008) Industrial food animal production, antimicrobial resistance, and human health, Annual Review of Public Health 29, 151-169.

[6] Wright, G. (2012) Antibiotic Resistome: A Framework Linking the Clinic and the Environment, In Antimicrobial Resistance in the Environment (Keen, P., and Montforts, M., Eds.), John Wiley \& Sons, Inc., Hoboken, New Jersey.

[7] Levy, S. (2012) Introduction. Antimicrobial Resistance in the Environment, In Antimicrobial Resistance in the Environment (Keen, P., and Montforts, M., Eds.), John Wiley \& Sons, Inc., Hoboken, New Jersey.

[8] Voolaid, V., Joers, A., Kisand, V., and Tenson, T. (2012) Co-occurrence of resistance to different antibiotics among aquatic bacteria, BMC Microbiology 12, 225.

[9] Roberts, M. (2012) Mechanisms of Bacterial Antibiotic Resistance and Lesions Learned from Environmental Tetracycline-Resistant Bacteria, In Antimicrobial Resistance in the Environment (Keen, P., and Montforts, M., Eds.), John Wiley \& Sons, Inc., Hoboken, New Jersey. 
[10] van Hoek, A. H., Mevius, D., Guerra, B., Mullany, P., Roberts, A. P., and Aarts, H. J. (2011) Acquired antibiotic resistance genes: an overview, Frontiers in Microbiology 2, 203.

[11] Couce, A., and Blazquez, J. (2009) Side effects of antibiotics on genetic variability, FEMS Microbiology Reviews 33, 531-538.

[12] Jensen, E. (2013) Microbiology Laboratory Manual (Biology 212), Biology Department of the College of Saint Benedict/St. John's University, Collegeville, MN.

[13] ASM. Chapter 67, In Manual of Clinical Microbiology (Murray, P., Ed.), ASM Press, Washington DC.

[14] Kirkman, T. (1996) Statistics to Use, http://www.physics.csbsju.edu/stats/ accessed July 2014

[15] Jorgensen, J., and Turnidge, J. (2003) Susceptibility Test Methods: Dilution and Disc Methods, In Manual of Clinical Microbiology (Murray, P., Ed.), ASM Press, Washington DC.

[16] Armstrong, J., Shigeno, D., Calomiris, J., and Seidler, R. (1982) Antibiotic-resistant Bacteria in Drinking Water, Applied and Environmental Microbiology 42, 277-283.

[17] Bell, J. B., Elliott, G. E., and Smith, D. W. (1983) Influence of sewage treatment and urbanization on selection of multiple resistance in fecal coliform populations, Applied and Environmental Microbiology 46, 227-232.

[18] McKeon, D., Calabrese, J., and Bissonnette, G. (1995) Antibiotic Restant Gram-negative Bacteria in Rural Groundwater Supplies, Water Research 29, 1902-1908.

[19] Barnhill, A. E., Weeks, K. E., Xiong, N., Day, T. A., and Carlson, S. A. (2010) Identification of multiresistant Salmonella isolates capable of subsisting on antibiotics, Applied and Environmental Microbiology 76, 2678-2680.

[20] Dantas, G., Sommer, M. O., Oluwasegun, R. D., and Church, G. M. (2008) Bacteria subsisting on antibiotics, Science 320, 100-103.

[21] Bennett, E. (1969) A study of the Distribution of Hetertrophic Bacteria in the Great Lakes, Bacteriology Branch of the Laboratories Division of the Ontario Water Resources Commission.

[22] Ash, R., Mauck, B., and Morgan, M. (2002) Antibiotic resistance of gram-negative bacteria in rivers, United States, Emerging Infectious Diseases 8, 713-716.

[23] Kelch, W. J., and Lee, J. S. (1978) Antibiotic resistance patterns of gram-negative bacteria isolated from environmental sources, Applied and Environmental Microbiology 36, 450-456.

[24] LeChevallier, M. W., Seidler, R. J., and Evans, T. M. (1980) Enumeration and characterization of standard plate count bacteria in chlorinated and raw water supplies, Applied and Environmental Microbiology 40, 922-930.

[25] Vaz-Moreira, I., Nunes, O. C., and Manaia, C. M. (2012) Diversity and antibiotic resistance in Pseudomonas spp. from drinking water, The Science of the Total Environment 426, 366-374.

[26] Guardabassi, L., Dalsgaard, A., and Olsen, J. E. (1999) Phenotypic characterization and antibiotic resistance of Acinetobacter spp. isolated from aquatic sources, Journal of Applied Microbiology 87, 659-667.

[27] Holmes, B., Snell, J. J., and Lapage, S. P. (1979) Flavobacterium odoratum: a species resistant to a wide range of antimicrobial agents, Journal of Clinical Pathology 32, 73-77.

[28] Projan, S. (2007) Genome Size Matters, Antimicrobial Agents and Chemotherapy 51, 1133-1134. 
[29] Touchon, M., Barbier, P., Bernardet, J. F., Loux, V., Vacherie, B., Barbe, V., Rocha, E. P., and Duchaud, E. (2011) Complete genome sequence of the fish pathogen Flavobacterium branchiophilum, Applied and Environmental Microbiology 77, 7656-7662.

[30] Peleg, A. Y., Seifert, H., and Paterson, D. L. (2008) Acinetobacter baumannii: emergence of a successful pathogen, Clinical Microbiology Reviews 21, 538-582.

[31] Sader, H. S., Streit, J. M., Fritsche, T. R., and Jones, R. N. (2004) Antimicrobial activity of daptomycin against multidrug-resistant Gram-positive strains collected worldwide, Diagnostic Microbiology and Infectious Disease 50, 201-204.

\section{ABOUT THE STUDENT AUTHOR}

Megan Bollin worked on this project in the summer of 2013 as part of her honors thesis in Biology at the College of Saint Benedict/Saint John's University. She graduated with a Bachelor of Arts degree in Biology in May 2014 and while an undergraduate spent a semester studying in London. She currently works for a biotechnology firm in the Twin Cities.

\section{PRESS SUMMARY}

The (over)use of antibiotics in agriculture and medicine over the last few decades has raised serious questions about the evolution of bacteria that have become resistant to standard antibiotics. In this paper, we sampled several local waterways in and around St. John's University in central Minnesota to determine if bacteria growing in these smaller, isolated areas had developed resistance to standard antibiotics and if so which classes of antibiotics were they now able to resist. The results indicate that bacteria growing in these areas are resistant to many common antibiotics. 